\title{
Characterization and frequency of a newly identified HIV-1 BF1 intersubtype circulating recombinant form in São Paulo, Brazil
} Sabri Saeed Sanabani ${ }^{1,2^{*}}$, Évelyn Regina de Souza Pastena ${ }^{1,2}$, Walter Kleine Neto ${ }^{1}$, Vanessa Pouza Martinez ${ }^{1,2}$,
Ester Cerdeira Sabino

\begin{abstract}
Background: HIV circulating recombinant forms (CRFs) play an important role in the global and regional HIV epidemics, particularly in regions where multiple subtypes are circulating. To date, several (>40) CRFs are recognized worldwide with five currently circulating in Brazil. Here, we report the characterization of near fulllength genome sequences (NFLG) of six phylogenetically related HIV-1 BF1 intersubtype recombinants (five from this study and one from other published sequences) representing CRF46_BF1.

Methods: Initially, we selected 36 samples from 888 adult patients residing in São Paulo who had previously been diagnosed as being infected with subclade F1 based on pol subgenomic fragment sequencing. Proviral DNA integrated in peripheral blood mononuclear cells (PBMC) was amplified from the purified genomic DNA of all 36blood samples by five overlapping PCR fragments followed by direct sequencing. Sequence data were obtained from the five fragments that showed identical genomic structure and phylogenetic trees were constructed and compared with previously published sequences. Genuine subclade F1 sequences and any other sequences that exhibited unique mosaic structures were omitted from further analysis
\end{abstract}

Results: Of the 36 samples analyzed, only six sequences, inferred from the pol region as subclade F1, displayed BF1 identical mosaic genomes with a single intersubtype breakpoint identified at the nef-U3 overlap (HXB2 position 9347-9365; LTR region). Five of these isolates formed a rigid cluster in phylogentic trees from different subclade F1 fragment regions, which we can now designate as CRF46_BF1. According to our estimate, the new CRF accounts for $0.56 \%$ of the HIV-1 circulating strains in São Paulo. Comparison with previously published sequences revealed an additional five isolates that share an identical mosaic structure with those reported in our study. Despite sharing a similar recombinant structure, only one sequence appeared to originate from the same CRF46_BF1 ancestor.

Conclusion: We identified a new circulating recombinant form with a single intersubtype breakpoint identified at the nef-LTR U3 overlap and designated CRF46_BF1. Given the biological importance of the LTR U3 region, intersubtype recombination in this region could play an important role in HIV evolution with critical consequences for the development of efficient genetic vaccines.

\section{Background}

The immense genetic variability of HIV-1 viruses is considered the key factor that frustrates efforts to halt the virus epidemic and poses a serious challenge to the development and efficacy of vaccines. Like other human positive-sense RNA viruses, HIV has a high mutation rate as a result of the error-prone nature of their reverse

\footnotetext{
* Correspondence: sabyem_63@yahoo.com

${ }^{1}$ Fundação Pro-Sangue, Hemocentro, São Paulo, Brazil
}

transcriptase $\left(3 \times 10^{-5}\right.$ mutations per nucleotide per replication cycle) $[1,2]$. This high rate of mutation coupled with the increased replication capacity of the virus $\left(10.3 \times 10^{9}\right.$ particles per day) [3], allows for the accumulation and fixation of a variety of advantageous genetic changes in a virus population, which are selected for by the host immune response and can resist newly evolving host defense. Recombination is another potential evolutionary source that significantly contributes to

\section{() Biomed Central}

(c) 2010 Sanabani et al; licensee BioMed Central Ltd. This is an Open Access article distributed under the terms of the Creative Commons Attribution License (http://creativecommons.org/licenses/by/2.0), which permits unrestricted use, distribution, and reproduction in any medium, provided the original work is properly cited. 
the genetic diversification of HIV by successfully repairing defective viral genes and by producing new viruses [4]. To date, HIV-1 viruses are classified into four phylogenetic groups: $\mathrm{M}, \mathrm{O}, \mathrm{N}$ and $\mathrm{P}$, which most likely reflect four independent events of cross-species transmission from chimpanzees [5-7]. The $M$ group (for main), responsible for the majority of viral infection worldwide, is further subdivided into nine subtypes (A$\mathrm{D}, \mathrm{F}-\mathrm{H}, \mathrm{J}$ and $\mathrm{K}$ ), among which subtypes $\mathrm{A}$ and $\mathrm{F}$ have been further classified into two sub-subtypes [5]. Moreover, early sequencing studies have provided evidence of recombination between genomes of different HIV subtypes $[8,9]$. Such interclade recombinant strains are consistently reported from regions where two or more clades are predominant. Recombinant strains from at least three unlinked epidemiological sources, which exhibit identical mosaic patterns, have been classified separately as circulating recombinant forms (CRFs) $[10,11]$. Currently, there are more than 40 defined CRFs http://www.hiv.lanl.gov that are epidemiologically important as subtypes [12]. In addition to the known CRFs, a large number of unique recombinant viruses, which are called unique recombinant forms (URFs), have been characterized worldwide [13]. Together, CRFs and URFs account for $18 \%$ of incident infections in the global HIV-1 pandemic [12]. HIV-1 subtypes, CRFs and URFs show considerably different patterns of distribution in different geographical regions [12,14].

In Brazil, the number of persons living with HIV reached an estimated number of 730,000 cases at the beginning of 2008 (2008 Report on the Global AIDS Epidemic). Like in other European countries and in North America, HIV-1 subtype B is a major genetic clade circulating in the country. However, the existence of other subtypes such as F1, C, B/C and B/F, has been consistently reported [15-23]. Data from recent studies of the near full length genomes (NFLG) of HIV have provided evidence of Brazilian CRF strains designated as CRF28_BF, CRF29_BF, CRF39_BF, CRF40_BF and CRF31_BC [17,24-26] http://www.hiv.lanl.gov/content/ sequence/HIV/CRFs/CRFs.html.

In 2006, Thompson and colleagues [27] published two NFLG of similar BF1 mosaic viruses from patients in Rio de Janeiro 94BR-RJ-41 (GenBank: AY455781) and 99UFRJ-16 (GenBank: AY455782). Here, we describe the HIV-1 NFLG of an additional six isolates with similar BF1 mosaic genomes from patients without evidence of direct epidemiological linkage.

\section{Methods}

\section{Study population}

The six samples reported in this study were from individuals residing in São Paulo in the southeast region of Brazil and considered the most populous city in South
Table 1 Characteristics of the six patients included in this study.

\begin{tabular}{|c|c|c|c|c|}
\hline Sample ID & Age/years & Sex & $\begin{array}{l}\text { CD4 count, } \\
\text { cells } / \mathrm{mm}^{2}\end{array}$ & $\begin{array}{l}\text { Viral load, } \\
\text { copies } / \mathrm{mL}\end{array}$ \\
\hline 06BR_FPS561 & 44 & $F^{1}$ & 81 & 721 \\
\hline 07BR_FPS625 & 35 & $\mathrm{~F}$ & 621 & 4734 \\
\hline 07BR_FPS742 & 47 & F & 140 & 123617 \\
\hline 07BR_FPS783 & 42 & $M^{2}$ & 209 & 80751 \\
\hline 07BR_FPS810 & 38 & $\mathrm{~F}$ & 208 & 49240 \\
\hline 07BR_FPS812 & 45 & $F$ & 362 & 5694 \\
\hline
\end{tabular}

F; Female, M; Male

America. The rationale for selection of these samples has been previously reported [28]. The data, including age, gender, number of CD4-positive T cells, and viral load were obtained from medical records and shown in Table 1. No evidence of direct epidemiological linkage could be established.

\section{Amplification and sequencing of HIV-1 DNA}

The genomic DNA used for the PCR analyses was extracted using the QIAamp blood kit (Qiagen) according to the manufacturer instructions. The NFLGs from five overlapping fragments were obtained by PCR using the Platinum Taq DNA polymerase (5 U/ $\mu \mathrm{l}$ ) (Invitrogen) and determined by a previously reported method $[16,17]$. To rule out the possibility of Taq-generated recombinants, an additional PCR product of $670 \mathrm{bp}$, which spans most of the viral LTR, was generated in separate PCR reactions using previously described primers and conditions [29]. All amplification reactions were done in duplicate to eliminate PCR artifacts, ensuring that sequenced NFLG were not assembled from heterogeneous DNA targets. To test for PCR carry over contamination, extraction and PCR negatives were run in each experiment. Both complementary DNA strands from each amplicon were directly sequenced by cycle sequencing using a variety of internal primers, BigDye terminator chemistry and Taq polymerase on an automated sequencer (ABI 3130, Applied Biosystems Inc., Foster City, CA), essentially according to the protocols recommended by the manufacturer. Fragments for each amplicon were assembled into contiguous sequences on a minimum overlap of $30 \mathrm{bp}$ with a $97-100 \%$ minimal mismatch and edited using the Sequencher program 4.7 (Gene Code Corp., Ann Arbor, MI).

\section{Screening for recombination events and identification of breakpoints}

Sequences were screened for the presence of recombination patterns by the jumping profile Hidden Markov Model (jpHMM) [30] and further confirmed using the bootscanning method [31] implemented by SimPlot 
3.5.1 for Windows [32]. The following parameters were used in this method: window size, $250 \mathrm{bp}$; step size, 20 bp; the F84 model of evolution (Maximum likelihood $(\mathrm{ML})$ ) as a model to estimate nucleotide substitution; transition $\mid$ transversion ratio, 2.0; and a bootstrap of 100 trees. In addition, the significant threshold for the bootscan was set at $90 \%$. The alignment of multiple sequences, including reference sequences representing subtypes A-D, F-H, J and K http://hiv-web.lanl.gov, were performed by the CLUSTAL X program [33] followed by manual editing in the BioEdit Sequence Alignment Editor program [34]. Gaps and ambiguous positions were removed from alignment. Positions of crossover sites were defined based on the distribution of informative sites supporting the two incongruent topologies that maximize the $\chi^{2}$ value [35], a method implemented in Simplot.

\section{Phylogenetic tree analysis}

Phylogenetic relationships between the individual sequence types were determined by two methods: the neighbor-joining (NJ) algorithm of MEGA v.4 [36] and the ML of PHYML v.2.4.4 [37]. For NJ, trees were constructed under the maximum composite likelihood substitution model and bootstrap resampling was carried out 1000 times for analysis by the MEGA software. ML phylogenies were constructed using the GTR + I + G substitution model and a BIONJ starting tree. Heuristic tree searches under the ML optimality criterion were performed using the NNI branch-swapping algorithm. The approximate likelihood ratio test (aLRT) based on a Shimodaira-Hasegawa-like procedure was used as a statistical test to calculate branch support. Comparison of tree topologies between subgenomic regions was performed using the algorithm described by Nye et al [38]. Trees were displayed using the program MEGA v.4 package. The nucleotide similarities were estimated using the maximum composite likelihood model implemented by MEGA v.4 software.

\section{GenBank accession numbers}

GenBank accession numbers for the proviral NFLG sequences reported in this study are (06BR_FPS561: HM026455, 07BR_FPS625: HM026456, 07BR_FPS742: HM026457, 07BR_FPS783: HM026458, 07BR_FPS810; HM026459, 07BR_FPS812: HM026460).

\section{Results}

\section{Recombinant Analysis}

A total of six strains (06BR FPS561, 07BR FPS625, 07BR FPS742, 07BR FPS783, 07BR FPS810, and 07BR FPS812) preliminarily classified as subclade $\mathrm{F} 1$ by sequence analysis of a partial pol region were corroborated by further phylogenetic analysis of the complete coding sequences and part of the LTR region. Analysis of the proviral NFLGs revealed all isolates retain intact reading frames for a majority of their genes and no gross deletions or rearrangements were observed. The NFLG sequence from each strain was initially investigated using jpHMM which showed them to display identical mosaic structures with a single intersubtype breakpoint identified at the nef-U3 overlap (HXB2 position 9347-9365). The recombinant genomes essentially consisted of subclades F1 and B as parental sequences. Fragments identified as subclade F1 were found to cover almost all of the genome coding regions while fragment classified as subtype $B$ consisted of a short sequence comprising the last part of the 3' LTR. Furthermore, the analysis also revealed that all the six isolates had a mosaic sequence pattern nearly identical to the previously published Brazilian BF1 isolates 94BR-RJ-41 (GenBank: AY455781) and 99UFRJ-16 (GenBank: AY455782). Based on these preliminary analyses, we reanalyzed all six sequences using the bootscanning method with three different subtype reference sequences (subtype B, F and C) obtained from the full-length alignment of the HIV sequence database http://hiv-web.lanl.gov. In agreement with the results obtained by jpHMM, bootscanning analysis confirmed similar mosaic structures with almost identical breakpoint positions within these six isolates (Figure 1). The BF1 intersubtype transitions were estimated at nucleotides 9347-9365, based on the HIV HXB2 numbering system, by mapping the informative site and $\chi^{2}$ maximization. To further test for recombination, ML phylogenetic trees were inferred for the regions of nucleotide sequence on either side of the breakpoints detected by bootscan method (Figure 1). This analysis corroborates the results from the bootscan and thus provided unambiguous evidence for a single recombination event supported by high aLRT values among the six isolates.

To rule out the possibility of Taq-generated recombinant artifacts, an additional PCR product of $670 \mathrm{bp}$ covering most of the viral LTR was generated in a separate PCR reaction using previously described primers and conditions [29]. The results confirmed the recombination breakpoint obtained using complete viral sequences.

\section{Phylogenetic analysis of regions bounded by the crossover sites}

As shown in Figure 2a, phylogenetic reconstructions for F1 specific regions bound by the crossover site, as defined by bootscan analysis, were compared with representatives of all subtype and sub-subtype references available in the HIV database (year 2008) and with other subclade F1 published sequences. The result of the ML tree revealed all our sequences clustered on a branch of subclade F1 and further into one separate sub-branch intrinsic to South America, particularly Brazil (100\% 


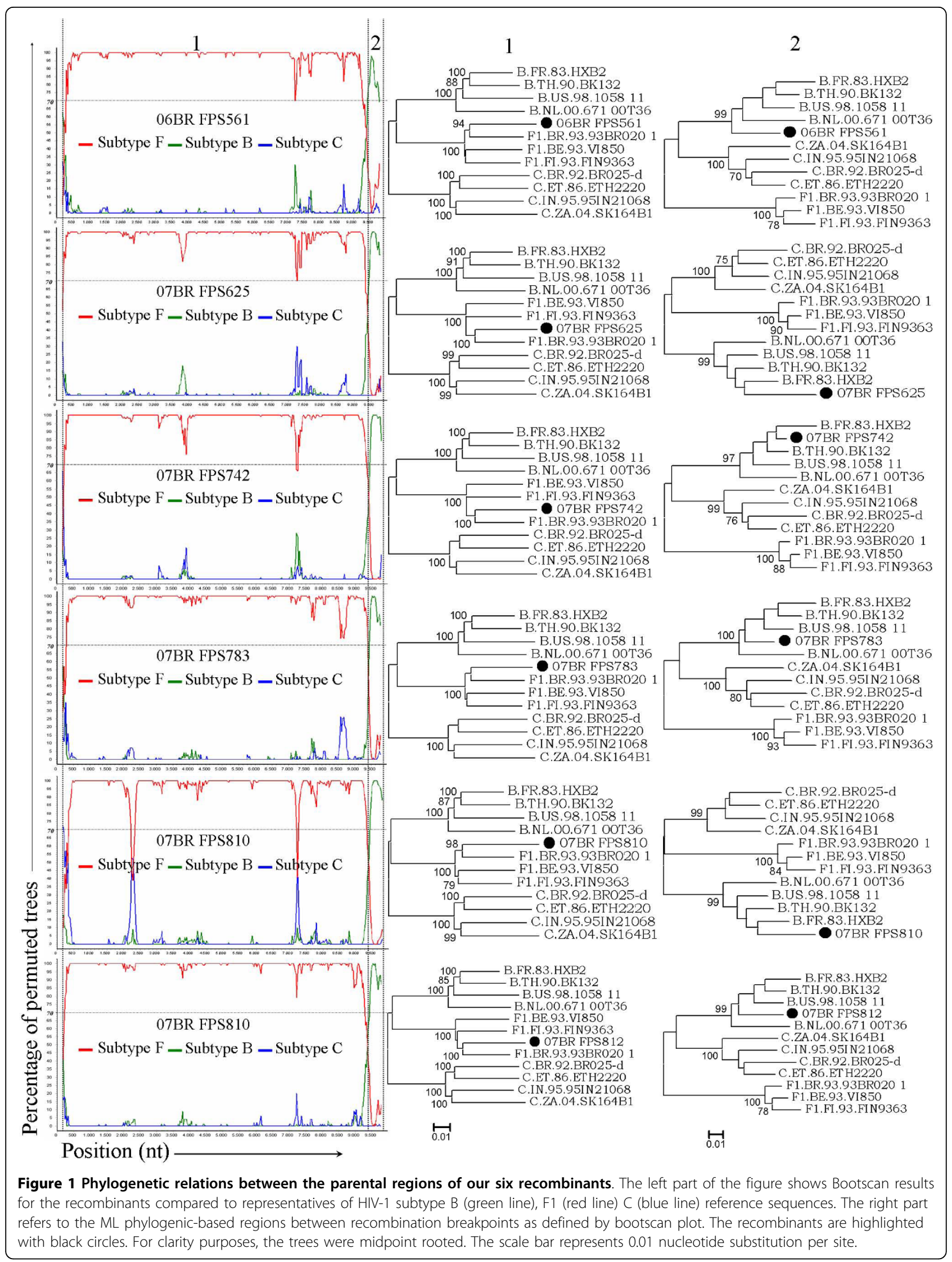




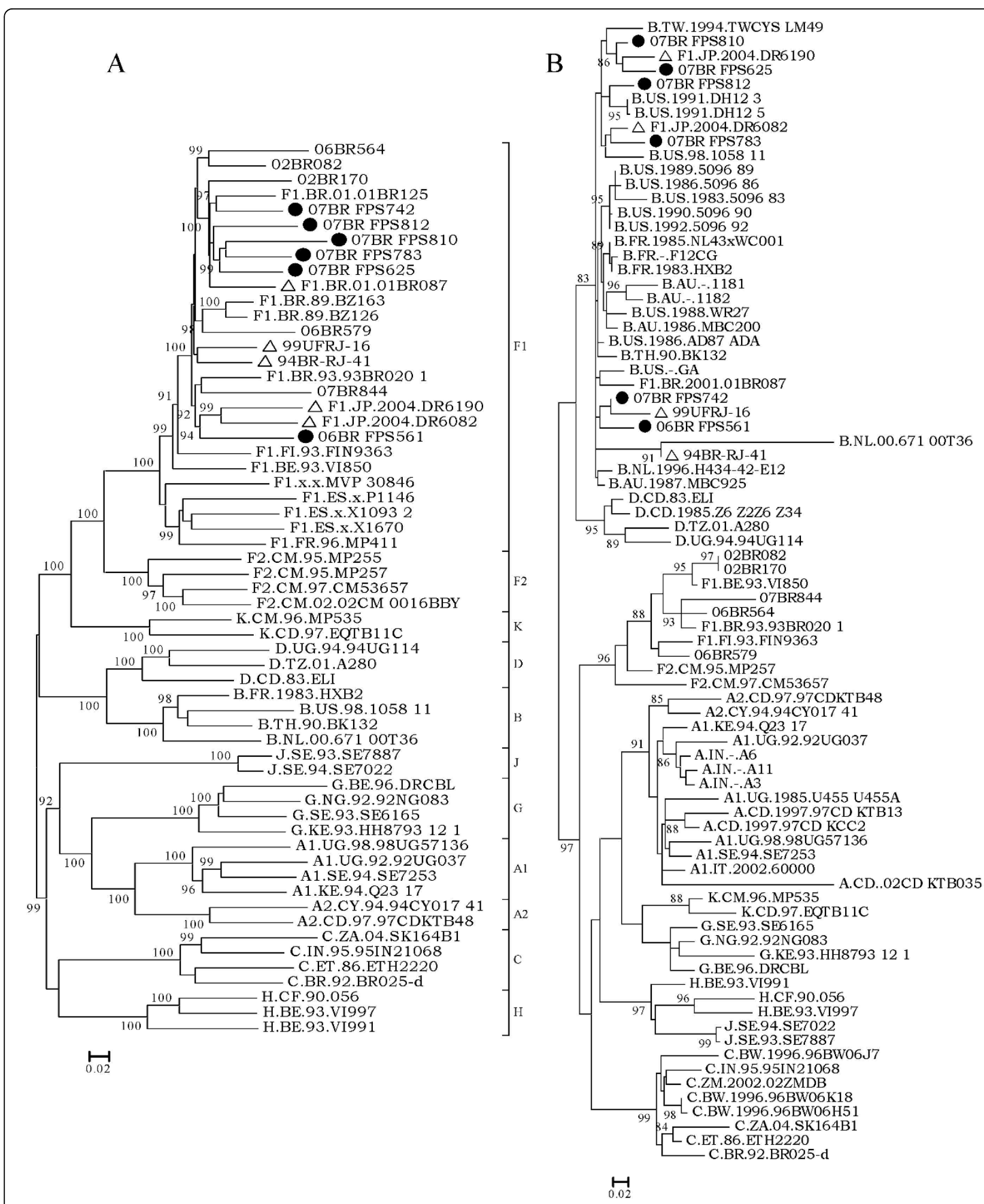

Figure 2 An exploratory ML tree calculated from fragments between breakpoints of sequences identified in this study (indicated by black circles), published sequences with identical breakpoints (indicated by triangle) and reference sequences of subtype A-D, F-H, J and K http://hiv-web.lanl.gov. (A) Tree of the viral genomes corresponding to the subclade F1 segments (HXB2 nucleotides 623-9347). (B) Tree of the viral genomes corresponding to the subtype B segments in the LTR region (HXB2 nucleotides 9348-9719). For clarity purposes, the tree was midpoint rooted. The approximate likelihood ratio test (aLRT) values of $\geq 90 \%$ are indicated at nodes. The scale bar represents 0.05 nucleotide substitution per site. 
aLRT). During analysis of the tree topology of the F1 region depicted in Figure 2a, all new sequences, except isolate 06BR FPS561, formed a single cluster with two previously published Brazilian isolates (F1. BR.01.01BR125 and F1.BR.01.01BR087) supported by $100 \%$ aLRT values. Isolate 06BR FPS561 formed a rigid subcluster (94\% aLRT) with two strains (F1.JP.2004. DR6190 and F1.JP.2004.DR6082) recently isolated in Japan and believed to be derived from Brazil [Tatsumi et al, unpublished study]. Additionally, the Brazilian isolates 94BR-RJ-41 and 99UFRJ-16 from Rio de Janeiro formed a separate branch $(<90 \%$ aLRT) distinct from the other branches. To test the stability and branching orders of the F1 fragment in our sequences, ML trees were independently made from gag-pol and env sequences using the same multiple genome alignment generated for the full length of F1 fragment (Figure 3a $\& 3 \mathrm{~b})$. The phylogenetic trees from both regions received an overall topological score of $78.5 \%$ according to the algorithm of Nye et al [38]. The computed topological score of the clusters that include all our isolates except 06BR FPS561 in both regions was 100\%. Isolate 06BR FPS561 placed the gag-pol region within the F1.JP.2004. DR6190, F1.JP.2004.DR6082, 07BR844 and 94BR-RJ-41 cluster with an $84 \%$ of aLRT value, while env grouped with another subcluster that included 06BR564 and 02BR082 (aLRT 94\%). The computed topological score of this cluster in both regions was 30\% with a branch length mismatch of $53.5 \%$. Similarly, isolates 94BR-RJ-41 and 99UFRJ-16 changed their topological positions over the gag-pol and env regions of their genomes (aLRT $<90 \%$ ). Thus, the shifting of topological positions of isolate 06BR FPS561, 94BR-RJ-41 and 99UFRJ-16 into two different phylogenetic trees is suggestive evidence of intrasubtype recombination event or other factors, such as convergence. Furthermore, the monophyletic cluster of isolates F1.JP.2004.DR6190 and F1.JP.2004.DR6082 depicted in Figure 2a was also supported in trees of both subgenomic regions (Figure $3 \mathrm{a} \& 3 \mathrm{~b}$ ).

The phylogenetic tree based on the fragment characterized as subtype B by bootscan from all of the six isolates is shown in Figure 2b. The resulting tree topology agrees with the accepted HIV-1 group M phylogeny and the majority of the internal nodes are supported with high aLRT values. Despite the fact that B fragments in these isolates have shorter sequences and some group $M$ variants cannot resolve some of the internal nodes, all of them can resolve the terminal nodes.

\section{Molecular rate of CRF46_BF1}

Five of the current six BF1 isolates described in this study (designated as CRF46_BF1 in the Los Alamos database) were detected in 36 samples selected from 888 samples infected with HIV-1 F1 based on pol subgenomic fragment sequencing [28]. Based on these results, the molecular distribution of the CRF46_BF1 accounts for $0.56 \%$ of the HIV-1 circulating strains in São Paulo.

\section{Identification of Related HIV-1 Strains in the database}

A search for similar recombination patterns in a sequence database revealed the occurrence of three isolates from Brazil (GenBank: AY455781; 94BR-RJ-41, AY455782; 99UFRJ-16 and DQ358801; 01BR087) and two isolates from Japan (GenBank: AB480299; F1. JP.2004.DR6082 and AB480301; F1.JP.2004.DR6190). It is to be noted that, as a result of our current analysis, the sequences F1.JP.2004.DR6082, F1.JP.2004.DR6190, and 01BR087, which are characterized as pure subclade F1 [17] [Tatsumi et al, unpublished study], showed strong phylogenetic evidence for recombination among subclade F1 and subtype B, suggesting that a revised classification of these isolates in the GenBank and the HIV databases is appropriate.

Next, we aimed to compare the recombinant profiles of our sequences to other HIV BF1 genomes at the nucleotide level to illustrate the distribution of their breakpoints. This was done by retrieving the full-length genomes from all BF1 and CRF_BF1 isolates available in the Los Alamos database. The automated jpHMM was used for mapping breakpoints with significant recombination signal (Figure 4). Our analysis showed that two variants (GenBank:DQ085869; BREPM11931 and DQ085870; BREPM11931) annotated as BF1 recombinants in the database, appear ancestral to subtype B strains. The recombination mapping of the nef-U3 overlap detected in our sequences was also found in CRF39_BF1 and four other URF BF1 recombinants. In addition, most of the sequences have undergone multiple rounds of recombination events. These data suggest that this part of the nef-U3 overlap is a possible 'hot spot' for recombination.

Fragment B from all six isolates shared 96\% sequence identity with the B stretch in the nef-U3 overlap from the Brazilian 93br029 which was isolated in 1993. Thus, we assume that the initial recombination event happened several years before 1993.

\section{Partial LTR nucleotides alignment features}

A detailed scrutinization of the partial nucleotide alignment of the 3' LTR regions relative to HXB2 and consensus sequences of other HIV subtypes (Year 2005) is shown in Figure 5. Conform to the consensus sequence GGGRNNYYCC, additional NF- $\kappa \mathrm{B}$ binding sites were found in three strains from the current study. A subclade F1 specific insert of 13-15 [39] nucleotides downstream of the NF- $\kappa B^{\mathrm{III}}$ binding site was not observed in our sequences and added further support to our results, 


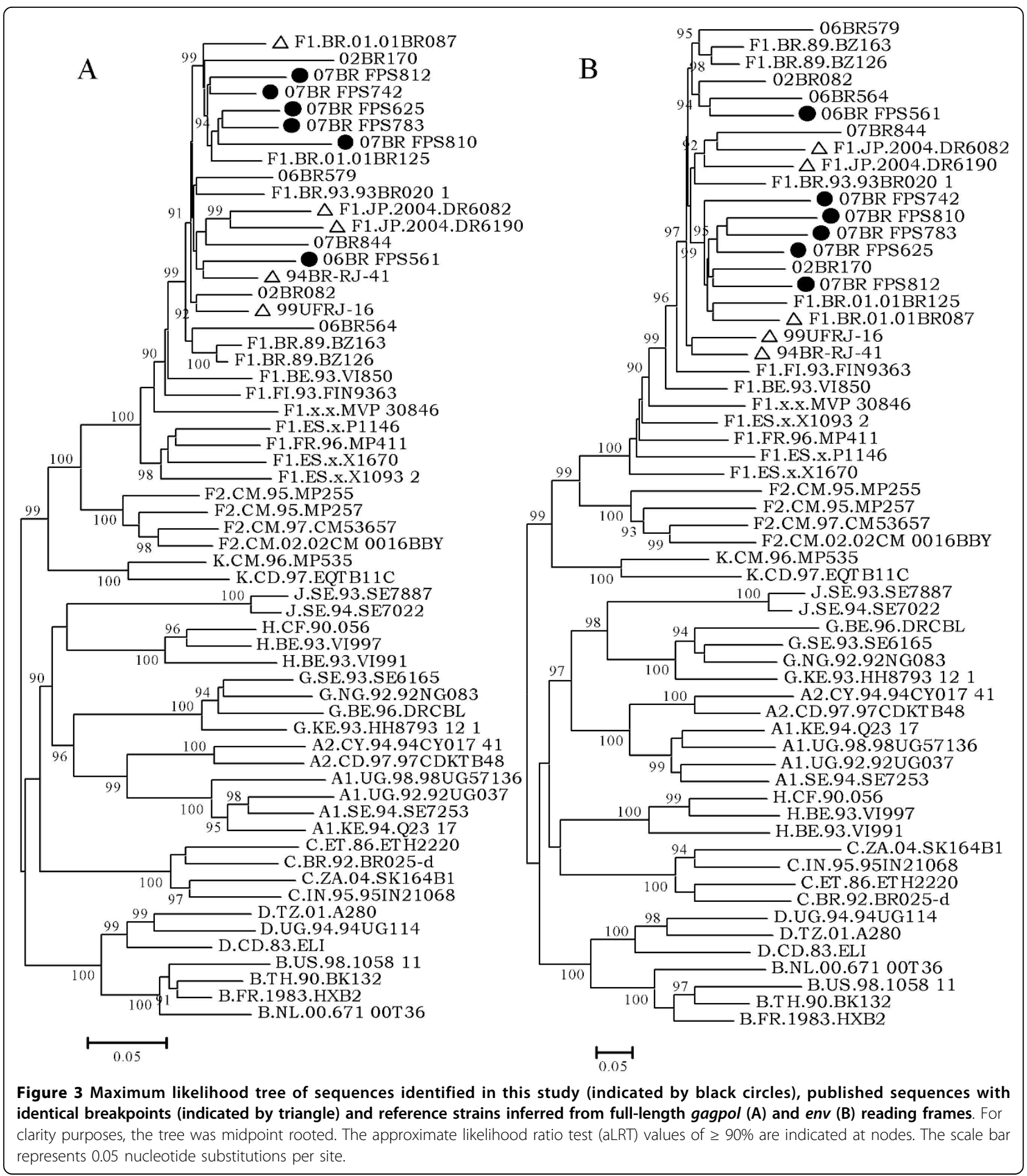

indicating that our sequences are not genuine F1 subsubtypes but BF1 recombinant isolates. Absence of this nucleotide signature was also observed in isolates F1. JP.2004.DR6082, F1.JP.2004.DR6190, and 01BR087, which have previously been classified as pure subclade F1 sequences.

\section{Discussion}

In the present study, we have characterized six NFLG sequences that posses mosaic genomic structure identical to the previously described strains, 94BR_RJ_41 and 99UFRJ_16 with a genome of predominantly subtype F1 and the nef-U3 overlap portion of the LTR of subtype B 


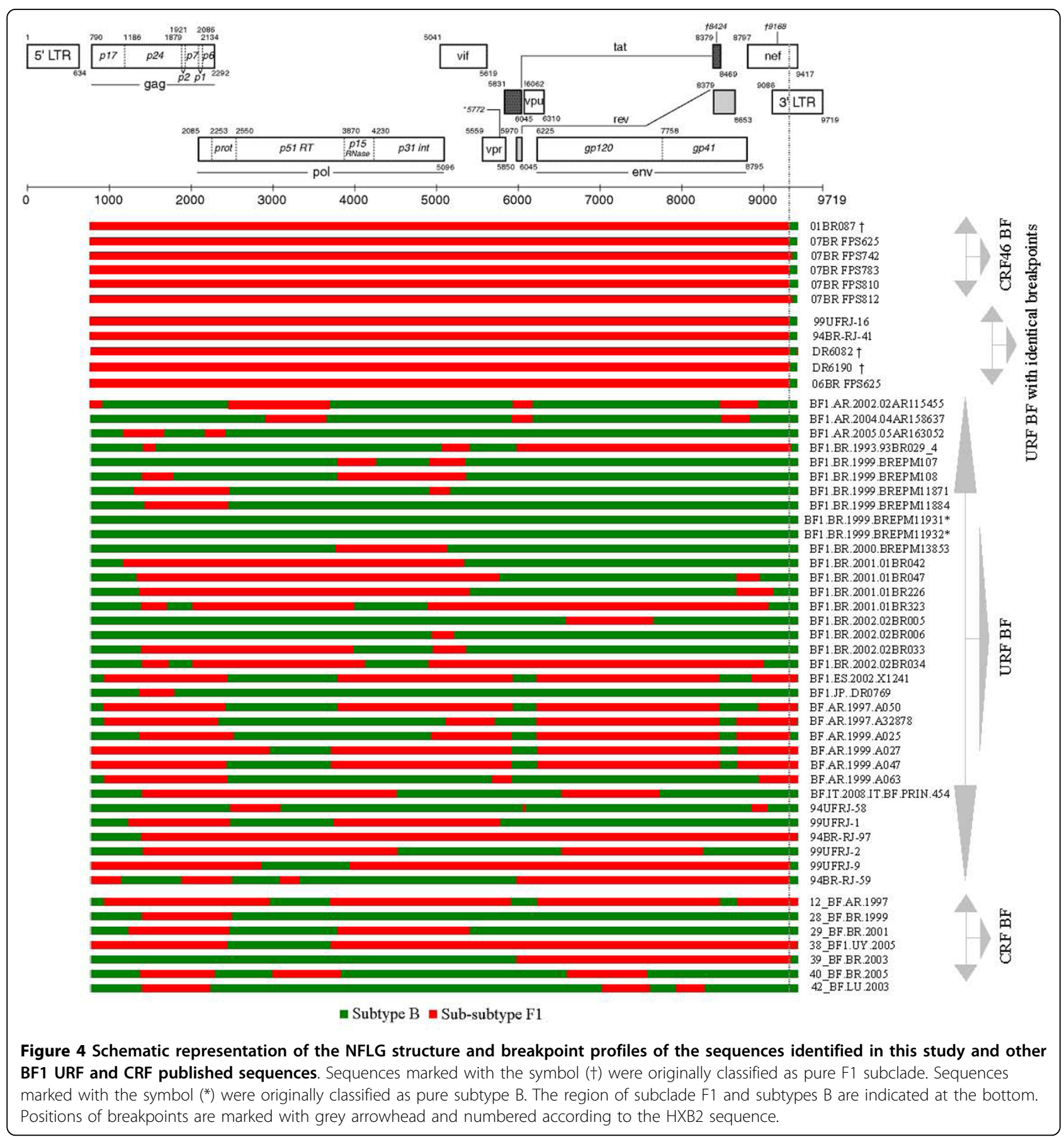

(Figure 1). Moreover, three additional full-length genome sequences, which were initially characterized as pure subclde F1, now clearly appear to harbor a small fragment derived from subtype B in their LTR in a position identical to the breakpoint reported in our sequences. In phylogenetic tree of the full length and subgenomic regions of F1 subclade segment, isolates F1. JP.2004.DR6082 F1.JP.2004.DR6190 (recovered from japanese patients), 94BR-RJ-41 and 99UFRJ-16 (recovered from patients residing in Rio de Janeiro) position outside the single cluster formed by isolates 01BR087 and all BF1 recombinants identified in this study, except 06BR FPS561 (recovered from patients residing in São Paulo) (Figure 2a\&3a). The discordant branching between gag-pol and env sequences of 06BR FPS561, 94BR-RJ-41 and 99UFRJ-16 isolates can be explained by the occurrence of another recombination events after the spread of their common ancestor. 


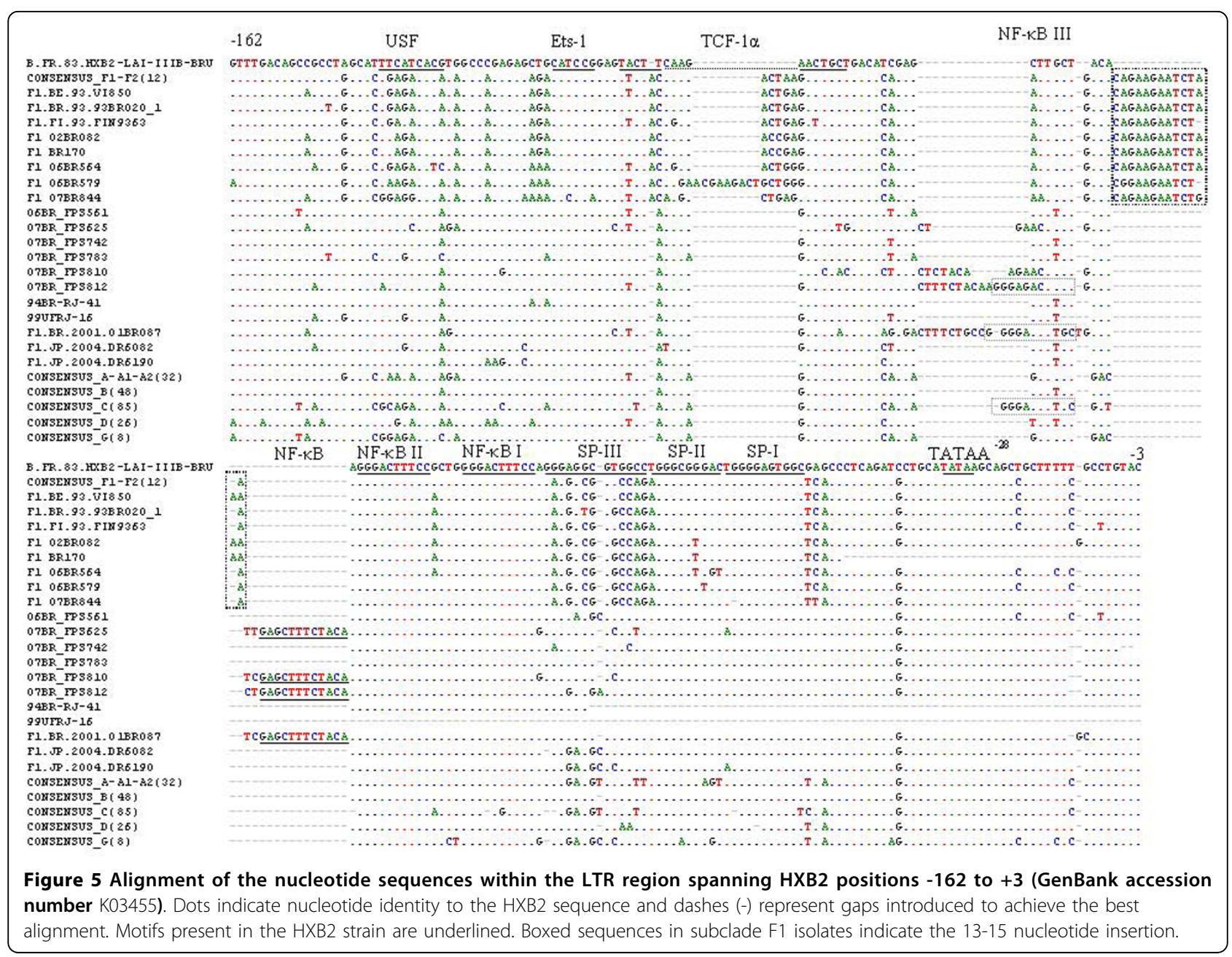

Generally, our results suggest that the 11 recombinant sequences were not the result of one, but at least three independent recombination events that produce similar simple recombinant structures. In particular, BF sequences isolated in Japan and Rio de Janeiro may have originated from different BF recombinant ancestors than those sequences isolated in São Paulo. Thus, by excluding all the isolates that branch out of the main cluster, we provide a total of 6 sequences (01BR087 and 5 sequences described in this study) that meet the formal requirement for assigning a new CRF46_BF1. Again, in the phylogenetic tree of the F1 subclade fragment, the two recently isolated Japanese strains (F1.JP.2004. DR6190 and F1.JP.2004.DR6082) formed a rigid subcluster with isolate 06BR FPS561 and branch outside the subcluster formed by the other five viruses described in this study, but still strongly position within the main Brazilian subclade F1 sequences. This result suggests that the viruses found in the Japanese patients share a distinct common ancestry originating in Brazil. It is possible that the heavy traffic of people from both countries across international borders could have facilitated the spread of these viruses in both countries.

Based on the criteria of inclusion of the samples in this study, we were able to show that the CRF46_BF1 accounts for $0.56 \%$ of the HIV-1 circulating strains in São Paulo, similar to the frequency of subclade F1 reported from this region [28]. The apparently low prevalence of the CRF46_BF is ecological and may not be due to inherent properties of the virus itself but rather to the chance results of subtype $B$ (a founder virus in Brazil), where it is introduced and consequently established into our HIV infected population before the new CRF and other subtypes are introduced.

Our analysis also showed that the recombination of subclade F1 with subtype B at the nef-U3 overlap portion of the LTR appears to be a recurrent finding because it has also been found in CRF39_BF1 and other unique HIV-1 recombinants $[17,25,40,41]$. In HIV, the existence of recombinational hot spots is common given that they have been described in cell-free systems [42] and exists in the dimer initiation sequence of the HIV-1 
5'-untranslated region and some preferential sites across the viral genome [43-46]. Several studies have demonstrated that RNA hairpin structures strongly correlate with recombination hotspots in various regions of the HIV-1 genome[42,43,46,47]. Thus, based on the later mechanisms, it is possible that hairpins promote recombination by hampering the RT during reverse transcription or direct interaction with template $[46,48,49]$.

The HIV-1 LTR region is composed of various cis-acting regulatory components needed for proviral DNA synthesis, integration of the nascent viral cDNA into the host cell genome, transcription and modulation of HIV genes expression [50,51]. Early reports showed that the LTR region is made up of three segments designated as U3, R and U5 [52]. The U3 modulatory region entirely overlaps with nef [53] and is essentially required during reverse transcription for first template transfer and integration of the provirus into the host genome. Moreover, this region seems to regulate the transcription pathway of HIV viral promoters by directly or indirectly interacting with a large number of cellular proteins, including NF-AT, Ets-1, USF, AP-1, COUP and Sp1 [54]. Thus, substitution through recombination of the nef-U3 overlap portion of the LTR with that of a genetically different subtype, as in our isolates, may affect the binding of both cellular and viral transcription factors. In turn, this may influence viral transcription levels, potentially enhancing the propagation of a recombinant virus leading to the persistance of a circulating form.

Several studies reported successful results in inhibiting HIV-1 replication by using synthetic siRNAs targeting either viral RNA sequences or cellular mRNAs encoding proteins that are critical for HIV-1 replication [55-58]. The study conducted by Yamamoto and his colleagues [59] showed a considerable sustainable suppression of HIV replication and control of CC-chemokine production associated with nef expression in HIV-1-infected macrophages following transfection of short hairpin RNA (shRNA) by a lentivirus vector system expressing HIV-specific shRNAs. These results allowed the authors to conclude that lentivirus-vector-based RNA interference of the U3-overlapping region of HIV-1 nef may have potential usefulness as a genetic vaccine against HIV-1 infection. Furthermore, Ludwig and collaborator [60] proved that HIV-1 contains an antisense gene in the U3-R regions of the LTR responsible for both an antisense RNA transcript and proteins. This antisense transcript has tremendous potential for intrinsic RNA regulation because of its overlap with the beginning of all HIV-1 sense RNA transcripts by 25 nucleotides. The novel HIV antisense proteins encoded in a region of the LTR that has already been shown to be deleted in some
HIV-infected long-term survivors and represent new potential targets for vaccine development $[60,61]$.

Given the biological relevance described to the U3 region, it is probable that the intersubtype recombination in this region could play an important role in HIV evolution with critical consequences for the development of efficient genetic vaccines.

During phylogenetic analysis, the B fragments of our six strains and the other five strains (marked with a triangle symbol in Figure 2b), which showed identical mosaic genomic structures, were clearly distinct from available South American subclade F1 sequences, particularly of Brazilian origin. This result coupled with the absence of the 13-15 nucleotides insertion downstream of the NF- $\kappa \mathrm{B}^{\mathrm{III}}$ binding site, which is typical for subclade F1, agrees with the interpretation that the segment at the nef-U3 overlap portion of the LTR of the eleven isolates originates from subtype B. Unlike the marked clustering of the eleven isolates in the tree generated from the F1 fragment, the tree of fragment B depicted in Figure $2 b$ shows them to fall in different subbranches within subtype B reference sequences. This result is most likely explained by the short lengths of the fragment B sequences.

\section{Conclusion}

In this study, we describe the NFLG sequence analysis from six HIV-1 isolates sampled from São Paulo and five other published isolates that had an identical breakpoints between subclades $\mathrm{F} 1$ and $\mathrm{B}$ at the nef-U3 overlap portion of LTR. Six of these sequences (five from this study and one from other published sequences) are currently classified as a member of the CRF46_BF1 family. Our data is relevant to guide diagnosis and vaccine development. We conclude that recombination is a potentially important mechanism that significantly contributes to HIV genetic variability with serious implications for diagnosis, drug treatment and optimal vaccine development.

\section{Acknowledgements}

This work was supported by grants 06/50096-0, 2004/15856-9 and 2007/ 04890-0 from the Fundação de Amparo a Pesquisa do Estado de São Paulo (FAPESP).

\section{Author details \\ 'Fundação Pro-Sangue, Hemocentro, São Paulo, Brazil. ${ }^{2}$ Retrovirology Laboratory, Federal University of São Paulo, Brazil.}

\section{Authors' contributions}

SS conceived and designed the study, did the data analysis of the sequences, and wrote the manuscript. ÉRP, WKN and VPM conducted the characterization of the full-length genome analysis. ECS designed, wrote the manuscript and directed the study. All authors read and approved the final manuscript. 


\section{Competing interests}

The authors declare that they have no competing interests.

Received: 28 January 2010 Accepted: 16 April 2010

Published: 16 April 2010

\section{References}

1. Mansky LM: The mutation rate of human immunodeficiency virus type 1 is influenced by the vpr gene. Virology 1996, 222:391-400.

2. Mansky LM, Temin HM: Lower in vivo mutation rate of human immunodeficiency virus type 1 than that predicted from the fidelity of purified reverse transcriptase. J Virol 1995, 69:5087-5094.

3. Perelson AS, Neumann AU, Markowitz M, Leonard JM, Ho DD: HIV-1 dynamics in vivo: virion clearance rate, infected cell life-span, and viral generation time. Science 1996, 271:1582-1586.

4. Worobey M, Holmes EC: Evolutionary aspects of recombination in RNA viruses. J Gen Virol 1999, 80(Pt 10):2535-2543.

5. Robertson DL, Anderson JP, Bradac JA, Carr JK, Foley B, Funkhouser RK, Gao F, Hahn BH, Kalish ML, Kuiken C, et al: HIV-1 nomenclature proposal. Science 2000, 288:55-56.

6. Gao F, Bailes E, Robertson DL, Chen Y, Rodenburg CM, Michael SF, Cummins LB, Arthur LO, Peeters M, Shaw GM, et al: Origin of HIV-1 in the chimpanzee Pan troglodytes troglodytes. Nature 1999, 397:436-441.

7. Plantier JC, Leoz M, Dickerson JE, De Oliveira F, Cordonnier F, Lemee V, Damond F, Robertson DL, Simon F: A new human immunodeficiency virus derived from gorillas. Nat Med 2009, 15:871-872.

8. Sabino EC, Shpaer EG, Morgado MG, Korber BT, Diaz RS, Bongertz V, Cavalcante S, Galvao-Castro B, Mullins JI, Mayer A: Identification of human immunodeficiency virus type 1 envelope genes recombinant between subtypes $B$ and $F$ in two epidemiologically linked individuals from Brazil. J Virol 1994, 68:6340-6346.

9. Robertson DL, Sharp PM, McCutchan FE, Hahn BH: Recombination in HIV1. Nature $1995,374: 124-126$.

10. Carr JK, Salminen MO, Albert J, Sanders-Buell E, Gotte D, Birx DL, McCutchan FE: Full genome sequences of human immunodeficiency virus type 1 subtypes $G$ and $A / G$ intersubtype recombinants. Virology 1998, 247:22-31.

11. Robertson DL, Anderson JP, Bradac JA, Carr JK, Foley B, Funkhouser RK, Gao F, BH H, Kalish ML, Kuiken C, et al: HIV-1 nomenclature proposal: a reference guide to HIV-1 classification. Human retroviruses and AIDS 1999: a compilation and analysis of nucleic acid and amino acid sequences. LOS Alamos, CA Kuiken CL, Foley B, Hahn B, et al 1999, 492-505.

12. Hemelaar J, Gouws E, Ghys PD, Osmanov S: Global and regional distribution of HIV-1 genetic subtypes and recombinants in 2004. AIDS 2006, 20:W13-23.

13. McCutchan FE: Global epidemiology of HIV. J Med Virol 2006, 78(Suppl 1): S7-S12.

14. Taylor BS, Sobieszczyk ME, McCutchan FE, Hammer SM: The challenge of HIV-1 subtype diversity. N Engl J Med 2008, 358:1590-1602.

15. Barreto CC, Nishyia A, Araujo LV, Ferreira JE, Busch MP, Sabino EC: Trends in antiretroviral drug resistance and clade distributions among HIV-1infected blood donors in Sao Paulo, Brazil. J Acquir Immune Defic Syndr 2006, 41:338-341.

16. Sanabani S, Neto WK, de Sa Filho DJ, Diaz RS, Munerato P, Janini LM, Sabino EC: Full-length genome analysis of human immunodeficiency virus type 1 subtype C in Brazil. AIDS Res Hum Retroviruses 2006, 22:171-176.

17. Sanabani S, Kleine Neto W, Kalmar EM, Diaz RS, Janini LM, Sabino EC: Analysis of the near full length genomes of HIV-1 subtypes $B, F$ and BF recombinant from a cohort of 14 patients in Sao Paulo, Brazil. Infect Genet Evol 2006, 6:368-377.

18. Passaes CP, Guimaraes ML, Bello G, Morgado MG: Near full-length genome characterization of HIV type 1 unique $B C$ recombinant forms from Southern Brazil. AIDS Res Hum Retroviruses 2009, 25:1339-1344.

19. Soares EA, Santos RP, Pellegrini JA, Sprinz E, Tanuri A, Soares MA: Epidemiologic and molecular characterization of human immunodeficiency virus type 1 in southern Brazil. J Acquir Immune Defic Syndr 2003, 34:520-526.

20. Brindeiro RM, Diaz RS, Sabino EC, Morgado MG, Pires IL, Brigido L, Dantas MC, Barreira D, Teixeira PR, Tanuri A: Brazilian Network for HIV
Drug Resistance Surveillance (HIV-BResNet): a survey of chronically infected individuals. Aids 2003, 17:1063-1069.

21. Rodrigues R, Scherer LC, Oliveira CM, Franco HM, Sperhacke RD, Ferreira JL, Castro SM, Stella IM, Brigido LF: Low prevalence of primary antiretroviral resistance mutations and predominance of HIV-1 clade $\mathrm{C}$ at polymerase gene in newly diagnosed individuals from south Brazil. Virus Res 2006, 116:201-207.

22. Brennan CA, Brites C, Bodelle P, Golden A, Hackett I Jr, Holzmayer V, Swanson $P$, Vallari $A$, Yamaguchi J, Devare $S$, et al: HIV-1 strains identified in Brazilian blood donors: significant prevalence of B/F1 recombinants. AIDS Res Hum Retroviruses 2007, 23:1434-1441.

23. Eyer-Silva WA, Couto-Fernandez JC, Morgado MG: Molecular epidemiology of HIV type 1 in inner Rio De Janeiro State, Brazil. AIDS Res Hum Retroviruses 2007, 23:303-308.

24. De Sa Filho DJ, Sucupira MC, Caseiro MM, Sabino EC, Diaz RS, Janini LM: Identification of two HIV type 1 circulating recombinant forms in Brazil. AIDS Res Hum Retroviruses 2006, 22:1-13.

25. Guimaraes ML, Eyer-Silva WA, Couto-Fernandez JC, Morgado MG: Identification of two new CRF_BF in Rio de Janeiro State, Brazil. AIDS 2008, 22:433-435.

26. Santos AF, Sousa TM, Soares EA, Sanabani S, Martinez AM, Sprinz E, Silveira J, Sabino EC, Tanuri A, Soares MA: Characterization of a new circulating recombinant form comprising HIV-1 subtypes $C$ and $B$ in southern Brazil. AIDS 2006, 20:2011-2019.

27. Thomson MM, Sierra M, Tanuri A, May S, Casado G, Manjon N, Najera R: Analysis of near full-length genome sequences of HIV type $1 \mathrm{BF}$ intersubtype recombinant viruses from Brazil reveals their independent origins and their lack of relationship to CRF12_BF. AIDS Res Hum Retroviruses 2004, 20:1126-1133.

28. Sanabani SS, Pastena ER, Kleine Neto W, Barreto CC, Ferrari KT, Kalmar EM, Ferreira S, Sabino EC: Near full-length genome analysis of low prevalent human immunodeficiency virus type 1 subclade F1 in Sao Paulo, Brazil. Virol J 2009, 6:78.

29. Gao F, Robertson DL, Morrison SG, Hui H, Craig S, Decker J, Fultz PN, Girard M, Shaw GM, Hahn BH, Sharp PM: The heterosexual human immunodeficiency virus type 1 epidemic in Thailand is caused by an intersubtype (A/E) recombinant of African origin. J Virol 1996, 70:7013-7029.

30. Schultz AK, Zhang M, Leitner T, Kuiken C, Korber B, Morgenstern B, Stanke M: A jumping profile Hidden Markov Model and applications to recombination sites in HIV and HCV genomes. BMC Bioinformatics 2006, 7:265.

31. Salminen MO, Carr JK, Burke DS, McCutchan FE: Identification of breakpoints in intergenotypic recombinants of HIV type 1 by bootscanning. AIDS Res Hum Retroviruses 1995, 11:1423-1425.

32. Lole KS, Bollinger RC, Paranjape RS, Gadkari D, Kulkarni SS, Novak NG, Ingersoll R, Sheppard HW, Ray SC: Full-length human immunodeficiency virus type 1 genomes from subtype C-infected seroconverters in India, with evidence of intersubtype recombination. J Virol 1999, 73:152-160.

33. Thompson JD, Gibson TJ, Plewniak F, Jeanmougin F, Higgins DG: The CLUSTAL_ $X$ windows interface: flexible strategies for multiple sequence alignment aided by quality analysis tools. Nucleic Acids Res 1997, 25:4876-4882.

34. Hall TA: BioEdit: a user-friendly biological sequence alignment editor and analysis program for windows 95/98/NT. Nucleic Acids SympSer 1999, 41:95-98.

35. Robertson DL, Hahn BH, Sharp PM: Recombination in AIDS viruses. J Mol Evol 1995, 40:249-259.

36. Tamura K, Dudley J, Nei M, Kumar S: MEGA4: Molecular Evolutionary Genetics Analysis (MEGA) software version 4.0. Mol Biol Evol 2007. 24:1596-1599.

37. Anisimova M, Gascuel O: Approximate likelihood-ratio test for branches: A fast, accurate, and powerful alternative. Syst Biol 2006, 55:539-552.

38. Nye TM, Lio P, Gilks WR: A novel algorithm and web-based tool for comparing two alternative phylogenetic trees. Bioinformatics 2006, 22:117-119.

39. Jeeninga RE, Hoogenkamp M, Armand-Ugon M, de Baar M, Verhoef K, Berkhout B: Functional differences between the long terminal repeat transcriptional promoters of human immunodeficiency virus type 1 subtypes A through G. J Virol 2000, 74:3740-3751. 
40. Gao F, Robertson DL, Carruthers CD, Morrison SG, Jian B, Chen Y, BarreSinoussi F, Girard M, Srinivasan A, Abimiku AG, et al: A comprehensive panel of near-full-length clones and reference sequences for nonsubtype B isolates of human immunodeficiency virus type 1. J Virol 1998, 72:5680-5698

41. Thomson MM, Delgado E, Herrero I, Villahermosa ML, Vazquez-de Parga $E$, Cuevas MT, Carmona R, Medrano L, Perez-Alvarez L, Cuevas L, Najera R: Diversity of mosaic structures and common ancestry of human immunodeficiency virus type $1 \mathrm{BF}$ intersubtype recombinant viruses from Argentina revealed by analysis of near full-length genome sequences. J Gen Virol 2002, 83:107-119.

42. Moumen A, Polomack L, Roques B, Buc H, Negroni M: The HIV-1 repeated sequence $\mathrm{R}$ as a robust hot-spot for copy-choice recombination. Nucleic Acids Res 2001, 29:3814-3821.

43. Balakrishnan M, Fay PJ, Bambara RA: The kissing hairpin sequence promotes recombination within the HIV-I 5' leader region. J Biol Chem 2001, 276:36482-36492.

44. Balakrishnan M, Roques BP, Fay PJ, Bambara RA: Template dimerization promotes an acceptor invasion-induced transfer mechanism during human immunodeficiency virus type 1 minus-strand synthesis. J Virol 2003, 77:4710-4721.

45. Magiorkinis G, Paraskevis D, Vandamme AM, Magiorkinis E, Sypsa V, Hatzakis A: In vivo characteristics of human immunodeficiency virus type 1 intersubtype recombination: determination of hot spots and correlation with sequence similarity. J Gen Virol 2003, 84:2715-2722.

46. Galetto R, Giacomoni V, Veron M, Negroni M: Dissection of a circumscribed recombination hot spot in HIV-1 after a single infectious cycle. J Biol Chem 2006, 281:2711-2720.

47. Galli A, Lai A, Corvasce S, Saladini F, Riva C, Deho L, Caramma I, Franzetti M, Romano L, Galli M, et al: Recombination analysis and structure prediction show correlation between breakpoint clusters and RNA hairpins in the pol gene of human immunodeficiency virus type 1 unique recombinant forms. J Gen Virol 2008, 89:3119-3125.

48. Moumen A, Polomack L, Unge T, Veron M, Buc H, Negroni M: Evidence for a mechanism of recombination during reverse transcription dependent on the structure of the acceptor RNA. J Biol Chem 2003, 278:15973-15982.

49. Roda RH, Balakrishnan M, Kim JK, Roques BP, Fay PJ, Bambara RA: Strand transfer occurs in retroviruses by a pause-initiated two-step mechanism. J Biol Chem 2002, 277:46900-46911.

50. Hiebenthal-Millow K, Greenough TC, Bretttler DB, Schindler M, Wildum S, Sullivan $J$ L, Kirchhoff F: Alterations in HIV-1 LTR promoter activity during AIDS progression. Virology 2003, 317:109-118.

51. Romanchikova N, Ivanova V, Scheller C, Jankevics E, Jassoy C, Serfling E: NFAT transcription factors control HIV-1 expression through a binding site downstream of TAR region. Immunobiology 2003, 208:361-365.

52. Varmus H: Retroviruses. Science 1988, 240:1427-1435.

53. Kirchhoff F, Greenough TC, Brettler DB, Sullivan JL, Desrosiers RC: Brief report: absence of intact nef sequences in a long-term survivor with nonprogressive HIV-1 infection. N Engl J Med 1995, 332:228-232.

54. Pereira LA, Bentley K, Peeters A, Churchill MJ, Deacon NJ: A compilation of cellular transcription factor interactions with the HIV-1 LTR promoter. Nucleic Acids Res 2000, 28:663-668.

55. Coburn GA, Cullen BR: Potent and specific inhibition of human immunodeficiency virus type 1 replication by RNA interference. J Virol 2002, 76:9225-9231.

56. Jacque JM, Triques K, Stevenson M: Modulation of HIV-1 replication by RNA interference. Nature 2002, 418:435-438.

57. Novina CD, Murray MF, Dykxhoorn DM, Beresford PJ, Riess J, Lee SK Collman RG, Lieberman J, Shankar P, Sharp PA: siRNA-directed inhibition of HIV-1 infection. Nat Med 2002, 8:681-686.

58. Qin XF, An DS, Chen IS, Baltimore D: Inhibiting HIV-1 infection in human T cells by lentiviral-mediated delivery of small interfering RNA against CCR5. Proc Natl Acad Sci USA 2003, 100:183-188.

59. Yamamoto T, Miyoshi H, Yamamoto N, Inoue J, Tsunetsugu-Yokota Y: Lentivirus vectors expressing short hairpin RNAs against the U3overlapping region of HIV nef inhibit HIV replication and infectivity in primary macrophages. Blood 2006, 108:3305-3312

60. Ludwig LB, Ambrus JL Jr, Krawczyk KA, Sharma S, Brooks S, Hsiao CB, Schwartz SA: Human Immunodeficiency Virus-Type 1 LTR DNA contains an intrinsic gene producing antisense RNA and protein products. Retrovirology 2006, 3:80.
61. Deacon NJ, Tsykin A, Solomon A, Smith K, Ludford-Menting M, Hooker DJ, McPhee DA, Greenway AL, Ellett A, Chatfield C, et al: Genomic structure of an attenuated quasi species of HIV-1 from a blood transfusion donor and recipients. Science 1995, 270:988-991.

doi:10.1186/1743-422X-7-74

Cite this article as: Sanabani et al:: Characterization and frequency of a newly identified HIV-1 BF1 intersubtype circulating recombinant form in São Paulo, Brazil. Virology Journal 2010 7:74.

\section{Submit your next manuscript to BioMed Central and take full advantage of:}

- Convenient online submission

- Thorough peer review

- No space constraints or color figure charges

- Immediate publication on acceptance

- Inclusion in PubMed, CAS, Scopus and Google Scholar

- Research which is freely available for redistribution

Submit your manuscript at www.biomedcentral.com/submit
Biomed Central 